\title{
1 Bildgebung bei Übergewichtigen
}

\author{
Uwe Keske
}

Die Bildgebung steht bei der Adipositas vor erheblichen Herausforderungen. Es ist mit einer teilweise deutlichen Verschlechterung der Bildqualität zu rechnen. Ferner können Adipöse für bildgebende Systeme zu schwer sein oder aufgrund ihrer körperlichen Dimensionen ggf. nicht untersucht werden. Daher müssen bei Adipösen bildgebende Verfahren gut geplant werden. Nach allgemeinen organisatorischen Erörterungen werden im Folgenden die spezifischen Probleme einzelner bildgebender Verfahren vorgestellt.

\subsection{Allgemeine Probleme}

Bereits der Patiententransport in die Radiologie kann eine Herausforderung darstellen. Das Vorhandensein von speziellen Transporteinrichtungen (Rollstühle oder Tragen in Spezialbreite) muss sichergestellt sein (Uppot et al. 2007). Natürlich kann das Patientengewicht ein Problem bedeuten. Während die Untersuchungstische von Geräten neuerer Bauart eine Tragkraft von $250-300 \mathrm{~kg}$ haben, liegt diese bei älteren Geräten nur bei 120-150 kg. Gelegentlich ist, insbesondere bei älteren Geräten, der Untersuchungstisch nur bis zu einem Wert deutlich unterhalb der maximalen Tragkraft zu verfahren; dies schränkt die Untersuchungsmöglichkeiten weiter ein. Ein zu hohes Patientengewicht kann Schaden am Gerät und so erhebliche Kosten sowie einen Geräteausfall verursachen. Zudem muss bei der Computertomografie (CT), Magnetresonanztomografie (MRT) und ggf. nu- klearmedizinischen Untersuchungen der Durchmesser der Gantry in der Planung berücksichtigt werden: Passt der Patient überhaupt ins Gerät? Der maximale Gantrydurchmesser liegt in der Regel zwischen 6o und $90 \mathrm{~cm}$, wobei am Unterrand noch ca. 15-18 cm für den Tisch abzuziehen sind (ein geringer GantryDurchmesser bietet übrigens bei allen Verfahren aufnahmetechnische Vorteile - ist aber bei der Untersuchung Adipöser nachteilig). Damit seitens der Radiologie eine optimale Untersuchungsplanung vorgenommen werden kann, sollte ein Gewicht von über $150 \mathrm{~kg}$ bereits bei der Anmeldung der Untersuchung mitgeteilt werden.

\subsection{Spezielle Probleme einzelner Bildgebender Verfahren}

Bei Röntgenaufnahmen führt die Zunahme des Körperdurchmessers zu einer Zunahme der Strahlenabsorption und der Streustrahlung. Hierdurch reduziert sich die Bildqualität bei steigender Strahlenexposition. Probleme gibt es insbesondere im Bereich des Körperstammes (Thorax, Abdomen, untere HWS sowie BWS und LWS). Abhilfe schafft eine Erhöhung der Aufnahmespannung $(\mathrm{kV})$ sowie eine Erhöhung des Röhrenstroms (mAs), wodurch allerdings die Strahlenexposition steigt (Yanch et al. 2009). Ferner muss bei Aufnahmen des Körperstammes berücksichtigt werden, dass Adipöse zu voluminös für das gängige 
Filmformat ( $35 \times 40 \mathrm{~cm})$ sein können. Hierbei spielen auch Vergrößerungseffekte durch die Zunahme des subkutanen Fettgewebes eine Rolle. In solchen Situationen sollten von vornherein mehr Aufnahmen als üblicherweise angefertigt werden, um das gesamte Gebiet abzudecken. Besonders betroffen von der Verschlechterung der Bildqualität sind Aufnahmen ohne Streustrahlenraster, z.B. auf der Intensivtherapiestation(s. Abb. 1). Raster sind teuer und auf der Intensivtherapiestation technisch schwierig einzusetzen. Bei Adipösen ist ihr Einsatz aber dennoch empfehlenswert. Keine bzw. nur geringe Limitationen gibt es bei Aufnahmen der Extremitäten.

Bei der Fluoroskopie (Durchleuchtung) kann es leicht passieren, dass, je nach Körperumfang, -gewicht und Geräteausstattung, die Grenzen des Möglichen überschritten werden. In solchen Fällen ist bei Bedarf auf die CT zurückzugreifen.

Beim Ultraschall kommt es durch die Absorption im subkutanen Fettgewebe zu erheblichen Limitationen. Zur Optimierung der Bildqualität sollte am Transducer die niedrigste mögliche Sequenz angewählt werden. Es sind individuelle Projektionen zu wählen, um Organe adäquat sichtbar zu machen. Damit individuelle Lagerungen erreicht werden können, sollten Lagerungshilfen wie z.B. Kissen verwendet werden.

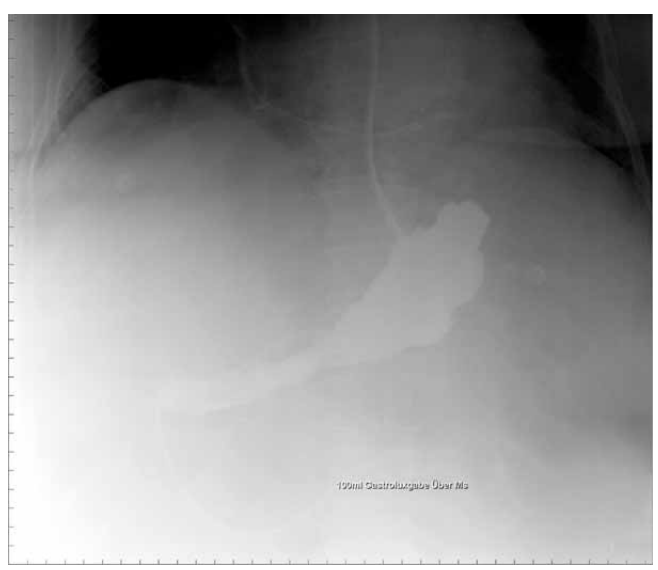

Abb. 1 72-jährige Patientin, Zustand nach Cholezystektomie, Körpergewicht $170 \mathrm{~kg}$. Postoperative Abdomenübersicht auf der Intensivtherapiestation, mit Kontrastmittel-Gabe über die Magensonde, angefertigt zum Ausschluss einer Perforation des Gastrointestinaltraktes, Speicherfolien-Aufnahme ohne Raster, deutlich suboptimale Bildqualität, kein Nachweis einer Perforation, sicherer Ausschluss derselben aber nicht möglich
Die Computertomografie hat in den letzten Jahren erhebliche Fortschritte bei der Datenakquisition und Bildberechnung gemacht. Moderne Geräte mit fortschrittlichen Detektoren (Multislice-CT) ermöglichen heute eine sehr gute Rauschunterdrückung und eine immer geringere Schichtdicke sowie multiplanare Rekonstruktionen in allen Rauschebenen. Die Bildgebung beim Adipösen wird hierdurch sehr wirkungsvoll unterstützt. Für Normalgewichtige wie für Adipöse gilt, dass der menschliche Körper im Stamm in der mediolateralen Richtung deutlich kaliberstärker ist als in der ventrodorsalen Richtung. Die Strahlenabsorption ist zudem in kraniokaudaler Richtung ungleich verteilt (im Thorax deutlich niedriger als im Oberbauch und im Becken deutlich höher als im Oberbauch). Eine automatisierte individuelle Anpassung des Röhrenstroms entsprechend dieser Notwendigkeiten (adaptive Dosismodulation) führt zu einer erheblichen Verbesserung der Bildqualität bei gleichzeitiger Erniedrigung der Strahlenexposition: Von ihr profitieren Adipöse besonders (s. Abb. 2 u. 3). Gleichzeitig verbessern moderne Bildberechnungsalgorithmen die Bildqualität maßgeblich. Seitens der Radiologie kann durch Erhöhung der Aufnahmespannung von zumeist 120 auf $140 \mathrm{kV}$ und durch Erhöhung des Röhrenstroms (mAs) sowie durch Verlängerung der Rotation von $0,5 \mathrm{~s}$ auf $1 \mathrm{~s}$ eine weitere Verbesserung der Bildqualität erreicht werden. Zusammenfassend gilt, dass Adipöse mittels eines modernen CT-Systems mit generell sehr akzeptabler Bildqualität untersucht werden können. $\mathrm{Zu}$ bedenken bleiben die einleitenden Anmerkungen zum Gantrydurchmesser und zum maximal zulässigen Tischgewicht. Eine „feet first“ Lagerung kann sich sowohl bei voluminösem Oberkörper als auch bei Platzangst vorteilhaft auswirken. Limitationen kann es immer durch ein infolge des großen Körperquerschnitts erhöhtes Rauschen geben. Wenn der Patient größer ist als das maximale sichtfeld (field of view, FOV), dann kann es zudem zu Aufhärtungsartefakten am Feldrand kommen, was die Bildqualität weiter beeinträchtigt. In der Regel ist die ergänzende Kontrastmittelgabe sinnvoll; hier kann es bei den klassischen Begleiterkrankungen Adipöser (Diabetes, Niereninsuffizienz) zu Problemen kommen.

Bei der Kernspintomografie sind die einleitenden Anmerkungen zum Gantrydurchmesser und zum maximal zulässigen Gewicht besonders zu berücksichtigen; die Limitationen sind hier größer als bei der CT. Generell ist das Signal-Rausch-Verhältnis bei Adipösen deutlich schlechter als bei Normgewichtigen. Wegen des großen Körperdurchmessers kann, um Einfaltungen zu vermeiden, eine Reduktion der 


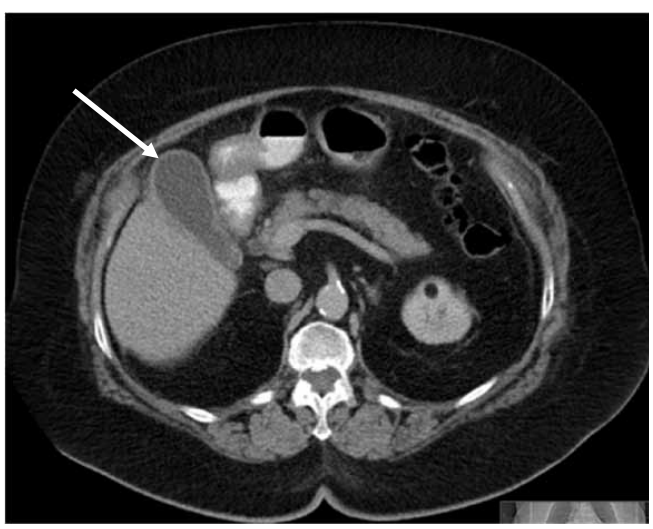

Abb. 2 Präoperative CT, transversale Aufnahme, sehr gute Bildqualität, Wandverdickung der Gallenblase bei Cholecystitis

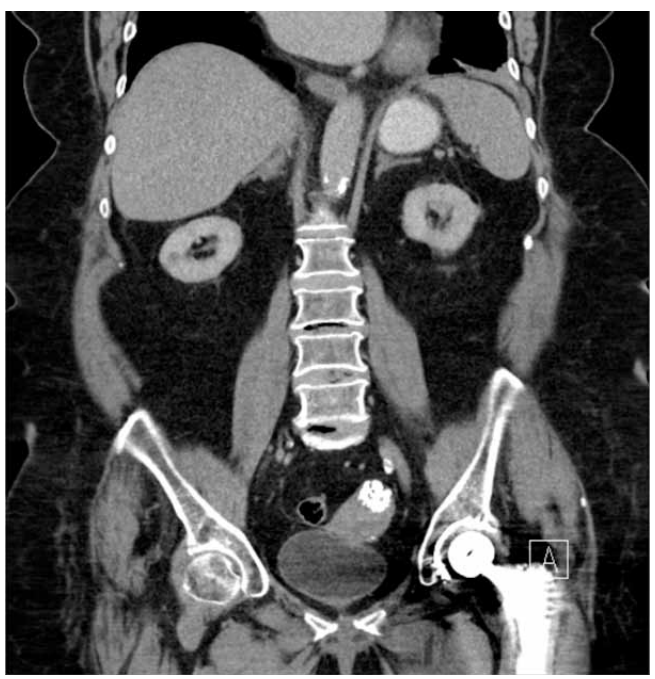

Abb. 3 Präoperative CT, koronare Rekonstruktion, sehr gute Bildqualität

räumlichen Auflösung nötig sein. Eine kurze Gantry wird zumeist besser toleriert (gerade bei Klaustrophoben), da Kopf und Hals bei den meisten Untersuchungen außerhalb der Gantry verbleiben können. Das offene MRT bietet bei der Untersuchung Adipöser Vorteile; diese Systeme sind jedoch in der Regel teurer und haben häufig eine schlechtere Bildqualität infolge eines niedrigeren Signal-Rausch-Verhältnisses und schwächerer Gradienten. Spezialgeräte für Adipöse mit herkömmlicher Bauart sind eben- falls verfügbar. Die früher gelegentlich genutzten Geräte der Veterinärmedizin sind heutzutage weniger einsetzbar, da diese zumeist nicht mit den für die Humanmedizin idealen Sequenzen ausgestattet sind.

In der Nuklearmedizin führt die verdickte Subkutis durch eine Absorption und Streuung der Photonen zu einer reduzierten Bildqualität. Zudem kann das Gewicht des Patienten die Untersuchung auf der Spezialtrage der Gammakamera unmöglich machen. Damit sind die „zusammengesetzten“ GanzkörperAufnahmen bzw. Schnittbildaufnahmen (SPECT) nicht möglich. Einzelaufnahmen im Patientenbett können aber durchgeführt werden. Insgesamt ist in der Regel die diagnostische Aussagekraft reduziert. Ggf. muss alternativ auf andere Verfahren zurückgegriffen werden (in der Regel MRT oder Computertomografie).

Auf der Intensivtherapiestation ist der Röntgen-Thorax unverzichtbar. Die Bildqualität liegt bei Adipösen deutlich unter der bei Normalgewichtigen; eine Alternative gibt es dennoch nicht. Um die aufnahmetechnischen Nachteile auszugleichen, ist es sinnvoll, zur Darstellung von zentralen Venenkathetern diese zuvor mit Kontrastmittel anzuspritzen. Weitere Aufnahmen auf der Intensivtherapiestation wie T-Drain-Darstellungen, Harnwegsdarstellungen und Magen-Darm-Darstellungen sind deutlich erschwert. Sie sollten vorrangig in der Röntgenabteilung durchgeführt werden, bei deutlich besseren Aufnahmebedingungen (Rasteraufnahmen auf dem Buckytisch, der speziell konstruierten Röntgenliege). Ein i.v. Urogramm ist bei extremem Übergewicht nicht erfolgversprechend durchführbar; stattdessen bietet sich ein CT an.

Die zur Abklärung des unklaren Abdomens herangezogenen Röntgenaufnahmen des Abdomens erbringen bei Adipositas nur wenige Ergebnisse. Hier ist die CT eindeutig zu favorisieren. In einem „One stop shopping“ kann neben der Diagnostik auch eine minimal-invasive Therapie eingeleitet werden (z.B. Drainagen bei Abszessen oder diagnostische Punktion von Flüssigkeitsverhalten). Prinzipiell ist beim CT die Strahlenexposition höher als bei konventionellen Aufnahmen. Jedoch ist hier zu beachten, dass bei Adipösen die mit Röntgenaufnahmen einhergehende Strahlenexposition erheblich sein kann. Für weitere Röntgenaufnahmen gilt, dass diese im Körperstamm mit Raster und unter bestmöglicher Einblendung durchgeführt werden sollten. Dies steigert die Bildqualität erheblich (s. Abb. 4).

Beim Polytrauma ist wegen der reduzierten Aussagekraft von Röntgenaufnahmen und Ultraschall 


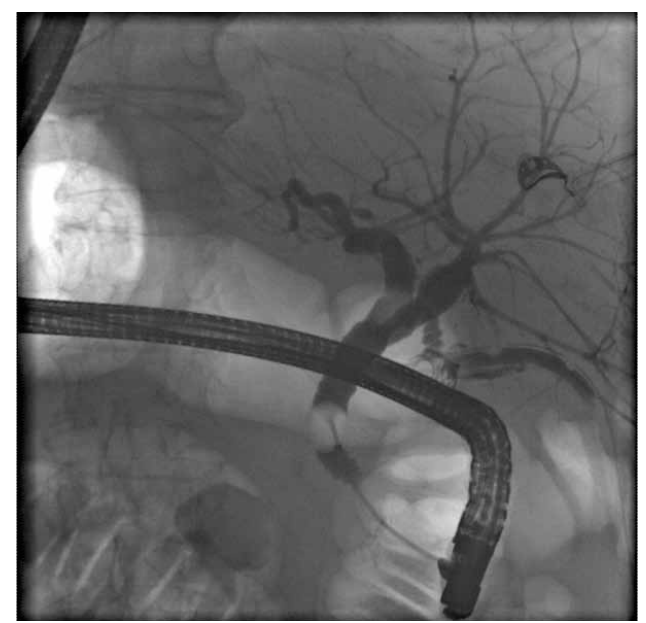

Abb. 4 Endoskopische retrograde Cholangiografie (ERC), durchgeführt in der Röntgenabteilung auf einem Durchleuchtungstisch mit Flachbilddetektor und Raster, gut eingeblendete Aufnahme, deutlich bessere Bildqualität als in Abbildung 1, Insuffizienz der Naht des Zysticusstumpfes

beim Vorhandensein einer entsprechenden Logistik auf die Trauma-CT zurückzugreifen. Hier kann mit einer Untersuchung die gesamte Abklärung erfolgen, vom Schädel über die Halswirbelsäule bis hin zu Thorax, Abdomen, Becken und Extremitäten (letztere mit dem Topogramm). Nach Erstversorgung sind dann im Bereich der Extremitäten ggf. Röntgenaufnahmen zu ergänzen.
Nach "gastric bypass surgery“ ist die wichtigste Untersuchung die Durchleuchtung. Diese ist häufig schwierig, wenn der Patient für das vorhandene Durchleuchtungsgerät zu schwer ist. Ist dies der Fall, so muss versucht werden, mit Bettaufnahmen zu arbeiten. Dies kann sowohl auf der Intensivtherapiestation als auch in der Radiologie erfolgen. Aufnahmen in der Radiologie können ggf. mit digitalen Detektoren durchgeführt werden, die Speicherfolien oder auch Kassetten mit Film-Folien-Kombinationen überlegen sind. Aufnahmen mit Raster bieten gegenüber Aufnahmen ohne Raster deutliche Vorteile. Bei nicht aussagekräftigen Untersuchungen sollte ernsthaft die Durchführung einer CT erwogen werden.

Bei Interventionen ist es besonders wichtig, dass bei der Untersuchungsanmeldung das Körpergewicht mitgeteilt wird. Es sind mehr Personal und spezielle Lagerungshilfen erforderlich. Ferner werden die Sedativa häufig nicht vom Patienten toleriert; eine Analgosedierung ist häufig erschwert oder kontraindiziert. Möglicherweise ist eine Narkose erforderlich, was zuvor eingeplant werden sollte.

\section{Literatur}

Uppot RN, Sahani DV, Hahn PF, Gervais D, Mueller PR (2007) Impact of obesity on medical imaging and image-guided intervention. AIR 188, 433-440

Yanch IC, Behrman RH, Hendricks MI, McCall JH (2009) Increased radiation dose to overweight and obese patients from radiographic examinations. Radiology 252, 128-139

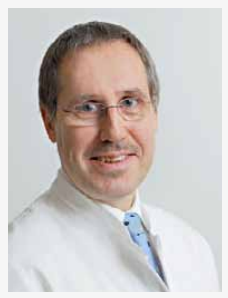

\section{PD Dr. med. Uwe Keske}

Nach Beendigung seines Medizinstudiums an der Georg-August-Universität Göttingen begann er seine Ausbildung zum Facharzt für Radiologie am Henriettenstift in Hannover. Fortführung der Facharztausbildungen Radiologie und Nuklearmedizin und wissenschaftliche Tätigkeit in Berlin am Universitätsklinikum Rudolf Virchow, Standorte Charlottenburg und Wedding (Direktor Univ.-Prof. Dr. Dr. h.c. R. Felix). Dort langjährige Oberarzt-Tätigkeit und Habilitation 1996. Seit 2001 Chefarzt Radiologie und Nuklearmedizin am Marienhospital Gelsenkirchen, seit 2007 auch in gleicher Funktion am Sankt Marien-Hospital Buer. 